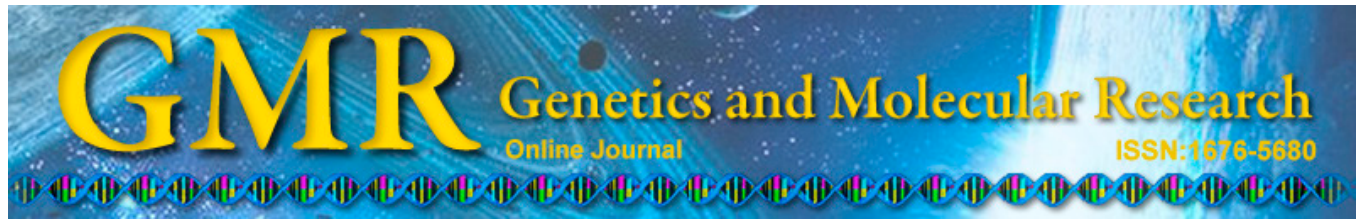

\title{
Identification of BPI protein produced in different expression system and its association with Escherichia coli F18 susceptibility
}

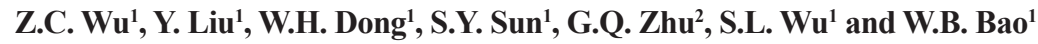 \\ ${ }^{1}$ College of Animal Science and Technology, Key Laboratory for Animal Genetics, \\ Breeding, Reproduction and Molecular Design of Jiangsu Province, \\ Yangzhou University, Yangzhou, China \\ ${ }^{2}$ College of Veterinary Medicine, Yangzhou University, Yangzhou, China \\ Corresponding author: W.B. Bao \\ E-mail:wenbinbao74@yahoo.com
}

Genet. Mol. Res. 14 (1): 1111-1123 (2015)

Received May 24, 2014

Accepted October 20, 2014

Published February 6, 2015

DOI http://dx.doi.org/10.4238/2015.February.6.15

ABSTRACT. The super antibiotic bactericidal/permeability-increasing (BPI) protein is a member of a new generation of proteins that have been implicated as endotoxin-neutralizing agents. In this study, recombinant porcine BPI protein was obtained by generating porcine BPI encoding prokaryotic, eukaryotic, and yeast expression vectors. Recombinant protein expression was detected in yeast GS115, Escherichia coli, and 293-6E cells by gel electrophoresis and Western blotting. Escherichia coli F18 is the primary Gram-negative bacteria in the gut and the main pathogen leading to diarrhea and edema disease in weaning piglets. Therefore, E. coli F18-resistant and -sensitive Sutai piglets were used to test differential expression of BPI protein by Western blotting and to investigate the potential correlation between BPI protein expression and E. coli F18-susceptibility. Recombinant porcine BPI protein expression was not detected in the prokaryotic and yeast expression systems; however, soluble protein was detected in the eukaryotic expression system. These data indicate the strong bacterio- 
static action of the BPI protein and confirm the feasibility of obtaining large amounts of recombinant porcine BPI recombinant protein using this eukaryotic expression system. In addition, the BPI protein expression levels in the E. coli F18-resistant group were significantly higher than those in the sensitive group, indicating that high BPI protein expression is associated with resistance to $E$. coli F18. Our findings provide a basis for further investigations into the development of a drug designed to confer resistance to $E$. coli $\mathrm{F} 18$ in weaning piglets.

Key words: Bactericidal/permeability-increasing protein; Porcine; Escherichia coli F18; Expression system

\section{INTRODUCTION}

Bactericidal/permeability-increasing protein (BPI) is a $55-\mathrm{kDa}$ endogenous cationic protein found in humans and other mammals. It is primarily present in the aniline blue particles of polymorphonuclear leukocytes, as well as on the surface of mononuclear leucocytes (Iovine et al., 1997; Canny and Levy, 2008). BPI not only kills Gram-negative bacteria (GNB) and neutralizes endotoxins (Akin et al., 2011), but also promotes complement activation and opsonization for increased phagocytosis, inhibits angiogenesis, inhibits the release of inflammatory mediators, and inhibits infection by fungi and protozoa. The function of BPI is related to its structure, which includes a cationic, lysine-rich N-terminus with antibacterial and lipopolysaccharide (endotoxin)-neutralizing activities (Iovine et al., 1997) and a C-terminus that contributes to the stability and opsonic activity (Ooi et al., 1991). BPI also plays an important role in natural defense mechanisms (Weiss et al., 1978; Iovine et al., 1997; Elsbach, 1998).

Previous studies have demonstrated that porcine $B P I$ gene polymorphisms affect disease resistance and susceptibility, and $B P I$ has been identified as a candidate gene for disease resistance breeding programs (Tuggle et al., 2003). The successful extraction and purification of porcine BPI protein from pig neutrophils was confirmed in both in vivo and in vitro biological activity experiments (Zhou et al., 1992, 1999). Furthermore, BPI protein was also shown to mediate endotoxin neutralization and Gram-negative antibacterial functions. Further studies showed that anti-human BPI protein antibodies enhanced the biological activity of porcine BPI protein in vitro (Zhou et al., 2002). Moreover, studies revealed the importance of BPI protein in the resistance to fungal and toxoplasma infections (Gavit and Better, 2000), indicating that this protein can be used to treat various diseases.

In this study, we constructed expression vectors encoding porcine BPI in prokaryotic, eukaryotic, and yeast expression vectors. The expression levels of the recombinant protein were analyzed by sodium dodecyl sulfate-polyacrylamide gel electrophoresis (SDS-PAGE) and Western blotting. The purified protein was used to investigate BPI function and evaluate methods for preparing porcine antimicrobial proteins on a large-scale. Escherichia coli F18 is the primary GNB in the gut and the primary pathogen leading to diarrhea and edema disease in weaned piglets. In previous studies, based on the FUT1 genetic marker, we established $E$. coli F18-resistant and -susceptible resource populations in Sutai pigs. Differential expression of BPI between enterotoxigenic E. coli F18 (ETEC F18)-resistant and ETEC F18-sensitive piglets was analyzed in our preliminary study, and we determined that expression of BPI is significantly high only in the duodenum and jejunum of pigs, suggesting that increased 
expression of BPI in the intestines is related to the resistance to ETEC F18 (Ye et al., 2011). Moreover, Zhu et al. (2013) observed significant differences between 35-day expression and expression during other development stages in the duodenum by analyzing the age-dependent tissue expression of porcine BPI in Sutai pigs (Zhu et al., 2013). The greatest susceptibility to $E$. coli $\mathrm{F} 18$ infection was observed at 35 days of age, with predominant $E$. coli F18 colonization in the duodenum. Because previous studies have indicated that the BPI gene is associated with $E$. coli $\mathrm{F} 18$, we investigated the role of recombinant porcine BPI protein in the resistance and susceptibility to E. coli $F 18$ infections in Sutai pigs.

\section{MATERIAL AND METHODS}

\section{Experimental materials}

DH5 $\alpha$ competent, BL21 (DE3) pLysS, and Rosetta cells were purchased from Invitrogen (Carlsbad, CA, USA). The pMD-18T vector was purchased from TaKaRa Biotechnology (Dalian, China). The pET22B, pPIC9, and pTT5 vectors, HEK293 cells, and Pichia GS115 were stored in our laboratory. Polymerase chain reaction (PCR) products were extracted using a gel extraction kit (BBI, Canada). Restriction enzymes (NcoI, XhoI, AvrII, and NotI) and T4 DNA ligase were purchased from TaKaRa Biotechnology. Platimum Pfx Polymerase and platinum HIFI Taq Polymerase were purchased from Invitrogen. The primers were synthesized by Shanghai Biological Engineering Technology (Shanghai, China). PCR products were sequenced using the ABI PRISM 377 DNA auto sequencer (Applied Biosystems, Foster City, CA, USA).

\section{Prokaryotic expression of the porcine BPI gene}

\section{Prokaryotic expression vector construction}

Single-stranded oligos for amplification of the porcine BPI gene were designed and synthesized according to the coding sequence available in the GenBank database (Accession No.: EF436278). The complete coding sequence was obtained by PCR amplification and ligated into the pMD-18T vector, which was then used to transform competent $E$. coli DH5 $\alpha$ cells. Moreover, mutation sites in the gene sequence were repaired by overlapping PCR. Positive clones were screened by sequencing and the resulting plasmid that included the correct objective sequence was designated as pMD-18T-BPI.

The pMD-18T-BPI plasmid was digested by $N c o$ I and $X h o$ I to yield a fragment of approximately 1450 -base pair fragment that was then ligated into the pET22B vector overnight at $16^{\circ} \mathrm{C}$ using T4 DNA ligase. The plasmid was then used to transform competent $E$. coli DH5 $\alpha$ cells, which were cultivated for 45 min prior to inoculation of $\mathrm{Amp}^{+} \mathrm{LB}$ solid culture plates containing ampicillin. Plates were cultured overnight at $37^{\circ} \mathrm{C}$. Finally, monoclonal colonies were verified by restriction enzyme digestion and sequencing; the correct vector was designated as pET22B-BPI1450.

\section{Optimization of conditions for porcine BPI gene expression in E. coli}

Following sequencing verification, pET22B-BPI1450 was used to transform E. coli BL21 and Rosetta cells. A monoclonal colony was used to inoculate $3 \mathrm{~mL}$ LB liquid medium 
containing $50 \mu \mathrm{g} / \mathrm{mL}$ Amp + . The culture was incubated overnight at $37^{\circ} \mathrm{C}$ with shaking at $230 \mathrm{rpm}$. Initial culture $(3 \mathrm{~mL})$ was diluted 1:100 and transferred to a new culture medium, and then cells were cultured for $110 \mathrm{~min}$ to an optical density (OD) ${ }_{600}$ value of 0.6-0.7. Next, $1 \mathrm{~mL}$ bacterial culture was used as a negative control, and protein expression was induced in the remaining culture by the addition of $1 \mathrm{mM}$ isopropyl $\beta$-D-1-thiogalactopyranoside and incubation for $4 \mathrm{~h}$ at $37^{\circ} \mathrm{C}$ and $16 \mathrm{~h}$ at $25^{\circ} \mathrm{C}$, respectively. Following centrifugation and removal of the supernatant, the bacterial pellet was resuspended in $50 \mu \mathrm{L}$ phosphate-buffered saline (PBS) and analyzed by SDS-PAGE.

\section{Eukaryotic expression of porcine BPI gene}

\section{Plasmid preparation}

The cDNA sequence of the porcine BPI gene was synthesized for codon optimization for mammalian expression in $2936 \mathrm{E}$ cells. The target DNA sequence containing an N-terminal Kozak sequence and a C-terminal 6X His tag was subcloned into the mammalian expression vector pTT5. Transfection grade bulk preparations of the plasmid were prepared (sterile, highly supercoiled, and endotoxin $<5 \mathrm{EU} / \mathrm{mg}$ ).

\section{Transient transfection and purification}

Cell cultures were harvested and centrifuged at $4000 \mathrm{rpm}$ ( $3220 \mathrm{~g}$ ) for $40 \mathrm{~min}$. The supernatant was collected and cells were stored at $-80^{\circ} \mathrm{C}$. The $\mathrm{pH}$ of the supernatant was adjusted to 8.0. Approximately $1.5 \mathrm{~mL}$ Ni Sepharose ${ }^{\mathrm{TM}} 6$ fast flow affinity chromatography resin was fully equilibrated with buffer A, added to the cell culture supernatant, and gently mixed for 5 $\mathrm{h}$. The resin was then collected in a syringe column and the target protein was step-gradient eluted with $50,100,250$, and $500 \mathrm{mM}$ and $1 \mathrm{M}$ imidazole solution. The target protein was mainly observed in the $500 \mathrm{mM}$ imidazole fraction.

\section{SDS-PAGE}

Prior to loading, $20 \mu \mathrm{L}$ sample was mixed with $5 \mu \mathrm{L} 5 \mathrm{X}$ loading buffer and heated at $90^{\circ} \mathrm{C}$ for $5 \mathrm{~min}$. The sample mixture was then loaded into the wells of a $12 \%$ SDS-PAGE gel (GenScript, Piscataway, NJ, USA). A pre-stained protein marker was loaded alongside the samples to identify the weight of protein bands in each sample. SDS-PAGE was performed using a Mini-PROTEAN Tetra system (Bio-Rad, Hercules, CA, USA). The gel was run at 120 $\mathrm{V}$ for $1500 \mathrm{~s}$ followed by $180 \mathrm{~V}$ for $2400 \mathrm{~s}$. The gel was then stained with $150 \mathrm{~mL}$ Coomassie brilliant blue R250 overnight at room temperature on a shaking table. Subsequently, the gel was washed and destaining until the background gel was clear.

\section{Western blot assay}

For Western blotting, the stacking gel was removed and the resolving gel was soaked in $1 \mathrm{~L}$ protein transfer buffer for $5 \mathrm{~min}$. Hybond-P polyvinylidene fluoride membranes and the filter paper were soaked in $100 \%(\mathrm{v} / \mathrm{v})$ methanol for $10 \mathrm{~s}$ and equilibrated in the protein transfer buffer for $5 \mathrm{~min}$. Band-transfer was carried out using a Trans-blot Electrophoretic Transfer 
Cell (Bio-Rad) at $100 \mathrm{~V}$ for $1 \mathrm{~h}$ at room temperature. Following transfer, non-specific binding sites on the membrane were blocked by incubation in 5\% dried skim milk in PBS containing Tween-20 for $1.5 \mathrm{~h}$. Membranes were then incubated with $0.2 \mu \mathrm{g} / \mathrm{mL}$ mouse-anti-His primary monoclonal antibody for $1.5 \mathrm{~h}$ at room temperature followed by incubation with the goat anti-mouse-horseradish peroxidase detection antibody (diluted 1:2500 in 5\% dried skim milk in PBS containing Tween-20) for $1 \mathrm{~h}$ at room temperature. The blot was rinsed 3 times with PBS containing Tween-20, incubated with luminescence substrate for $3 \mathrm{~min}$ and exposed to X-ray film for $60 \mathrm{~s}$.

\section{Yeast expression of the porcine BPI gene}

\section{Construction of the yeast expression vector}

The protein structure of the porcine BPI gene (accession No.: EF436278) was analyzed to identify the signal peptide sequence using the SignalP 3.0 software (http://www. cbs.dtu.dk/services/SignalP 3.0). Primers were designed for PCR amplification of the BPI cDNA sequence minus the signal sequence. The pMD-18T/BPI construct containing the BPI cDNA sequence was stored in our laboratory. The BPI sequence incorporating the AvrII/NotI restriction enzyme sites and a 6 XHis tag was amplified using pMD-18T/BPI as a template with the following primers designed using the Primer Premier 5.0 software (Premier Biosoft, Palo Alto, CA, USA): F: 5'-TCCGCCTAGGATGGCCAGGGGCGCTGACAAC-3', R: 5'-ATAAGAATGCGGCCGCTCAGTGGTGGTGGTGGTGGTGCTCGA-3'. The 50- $\mu$ L PCR reaction system consisted of $5 \mu \mathrm{L}$ 10X Accuprime Pfx Buffer mix, $5 \mu \mathrm{L}$ 10X Amplification enhancer, $1 \mu \mathrm{L}$ each $10 \mu \mathrm{M}$ primer, $0.5 \mu \mathrm{L}$ Accuprime $P f x$ polymerase, $1 \mu \mathrm{L}$ pMD-18T/BPI template, and $37.5 \mu \mathrm{L} \mathrm{ddH_{2 }} \mathrm{O}$. The PCR amplification program consisted of 1 cycle of predenaturation at $95^{\circ} \mathrm{C}$ for $3 \mathrm{~min}, 25$ cycles of denaturation at $95^{\circ} \mathrm{C}$ for $30 \mathrm{~s}$, annealing $60^{\circ} \mathrm{C}$ for 30 $\mathrm{s}$, and extension at $68^{\circ} \mathrm{C}$ for $80 \mathrm{~s}$; a final cycle of $68^{\circ} \mathrm{C}$ for $5 \mathrm{~min}$. PCR products were evaluated on $1 \%$ agarose gels stained with ethidium bromide. PCR products were extracted using a gel extraction kit (Bio Basic, Inc., Ontario, Canada). The complete AvrII-BPI-6XHis-NotI target fragment was digested with AvrII and NotI, ligated into the pPIC9 vector, and transformed into competent DH5 $\alpha$ cells. Positive colonies were picked and recombinant pPIC9-BPI-6XHis was extracted by alkaline lysis. The construct was verified by PCR amplification, enzyme digestion, and sequencing (Shanghai Biotechnology, Shanghai, China).

\section{Electroporation of GS115}

Monoclonal GS115 colonies were picked and cultivated at $30^{\circ} \mathrm{C}$ in $20 \mathrm{~mL}$ Yeast peptone dextrose medium. At an OD value of approximately 1.0, competent GS115 were prepared. The pPIC9-BPI-6XHis plasmid ( $200 \mathrm{ng} / \mu \mathrm{L} ; 50 \mu \mathrm{L})$ was digested with $5 \mu \mathrm{L}$ NotI, $50 \mu \mathrm{L} 10 \mathrm{X}$ H Buf-

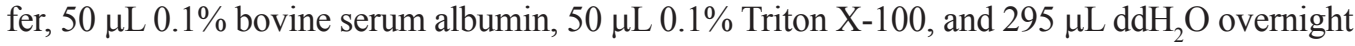
at $37^{\circ} \mathrm{C}$. The linearized plasmid was isolated by ethanol precipitation and dissolved in $20 \mu \mathrm{L}$ $\mathrm{ddH}_{2} \mathrm{O}$ and added to $200 \mu \mathrm{L}$ competent GS115 on ice for $10 \mathrm{~min}$. Finally, the pPIC9-BPI-6XHis plasmid was transfected into competent DH5 $\alpha$ cells by electroporation $(1680 \mathrm{~V})$.

\section{Induced protein expression}

Positive clones were cultured in $10 \mathrm{~mL}$ BMGY medium overnight at $30^{\circ} \mathrm{C}$ with shak- 
ing. Subsequently, the culture was centrifuged at $2500 \mathrm{rpm}$ for $5 \mathrm{~min}$ at $4^{\circ} \mathrm{C}$. After centrifugation, the thallus was used for heavy suspension culture in $10 \mathrm{~mL}$ BMGY medium. Protein expression was induced for 3 days by adding $100 \mu \mathrm{L}$ methanol into the medium at $24 \mathrm{~h}$ intervals. Expression of the target protein was detected by SDS-PAGE and Western blotting as described above.

\section{Differential expression of porcine BPI gene in $E$. coli F18-resistant and susceptible piglets}

This study was conducted in 3 E. coli F18-resistant piglets and 3 E. coli F18-susceptible piglets (Bao et al., 2012). All experiments were conducted in the Animal Hospital of Yangzhou University according to the regulations of the Administration of Affairs Concerning Experimental Animals (Ministry of Science and Technology, China, revised in June 2012) and approved by experimental animal using permit with No. SYXK (Su) 2012-0029. The animals were allowed access to feed and water ad libitum under normal conditions and humanely sacrificed as necessary to ameliorate suffering. Fifteen centimeters of the duodenum were obtained according to the method described by Alwan et al. (1998) to isolate and prepare intestinal epithelial cells. For nuclear translocation experiments, nuclear and cytosolic fractions were obtained using the NE-PER kit (Pierce, Rockland, IL, USA) according to the manufacturer protocol. Protein levels were normalized using the BCA kit (Pierce). SDS-PAGE was performed with $10 \mu \mathrm{L}$ protein separated on a $10 \%$ gel at $160 \mathrm{~V}$ for $70 \mathrm{~min}$. For Western blotting analysis proteins were transferred to polyvinylidene fluoride membranes and immunoblotted with antibodies to BPI (1:500, ABmart, Shanghai, China), $\beta$-actin, and anti-rabbit $(1: 3,000)$ IgG.

\section{RESULTS}

\section{Prokaryotic expression analysis}

SDS-PAGE analysis of bacterial samples following induction for $4 \mathrm{~h}$ at $37^{\circ} \mathrm{C}$ revealed a recombinant protein of approximately $57 \mathrm{kDa}$ that was absent in the pre-induction culture (Figure 1). Expression of the 57-kDa protein was also absent from cultures induced for $16 \mathrm{~h}$ at $25^{\circ} \mathrm{C}$ (Figures 2).

\section{Yeast expression analysis}

Successful construction of pPIC9-BPI-6XHis was confirmed by sequencing. However, inducible expression of the target protein was not detected by SDS-PAGE and Western blotting analyses (Figures 3 and 4).

\section{Eukaryotic expression analysis}

The recombinant eukaryotic expression vector encoding BPI was transiently transfected into HEK293 cells and the target protein was isolated from the cell culture supernatant by 1-step affinity chromatography. Analysis of the eluted fractions by SDS-PAGE and Western blot revealed the presence of the BPI protein with an apparent molecular weight of $62 \mathrm{kDa}$ 


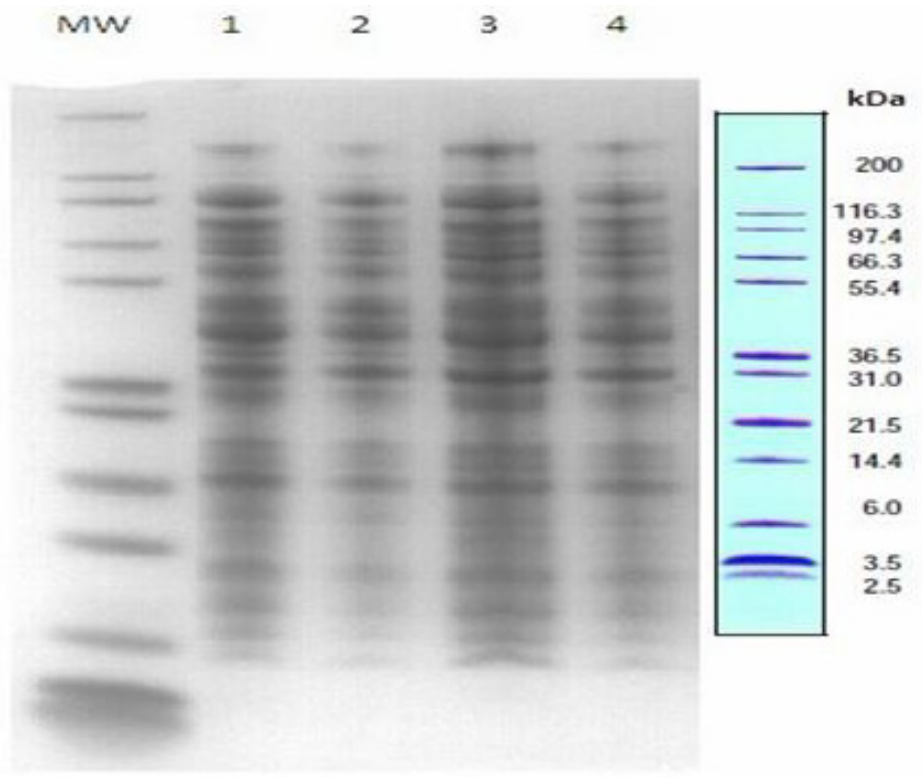

Figure 1. SDS-PAGE analysis of bacterial samples following induction for $4 \mathrm{~h}$ at $37^{\circ} \mathrm{C}$. Lane $M W$ : molecular weight; lane 1: pre-induction with BL21(DE3)plysS; lane 2: post-induction with BL21(DE3)plysS; lane 3 preinduction with Rosetta(DE3); lane 4 represents post-induction with Rosetta(DE3).

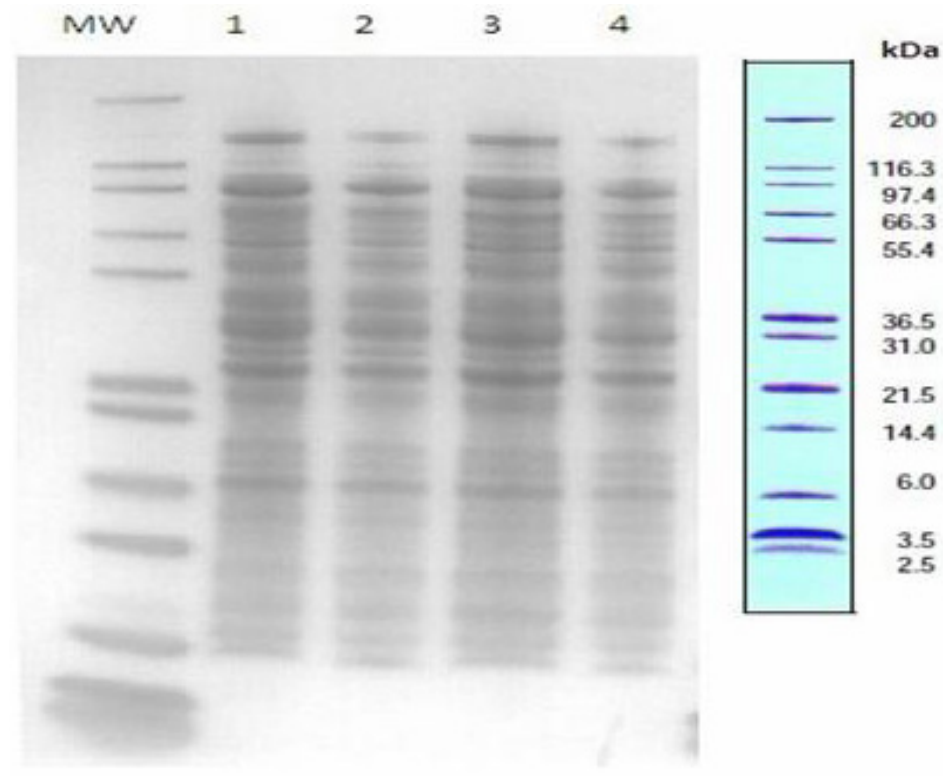

Figure 2. SDS-PAGE analysis of bacterial samples following induction for $16 \mathrm{~h}$ at $25^{\circ} \mathrm{C}$. Lane $M W$ : molecular weight; lane 1: pre-induction with BL21(DE3)plysS; lane 2: post-induction with BL21(DE3)plysS; lane 3 preinduction with Rosetta(DE3); lane 4 represents post-induction with Rosetta(DE3). 


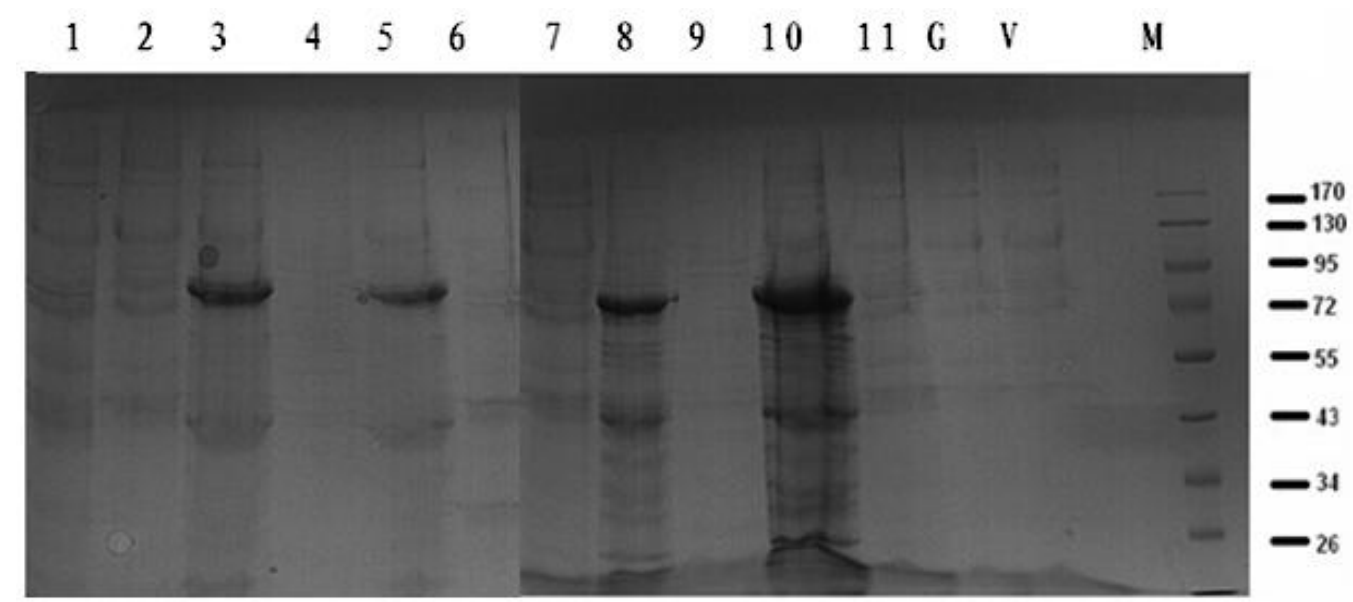

Figure 3. SDS-PAGE analysis of porcine BPI expression in yeast GS115. Lane M represents protein marker; lanes 1-11 represents recombinant porcine BPI protein; G represents yeast GS115; V represents pPIC vector.

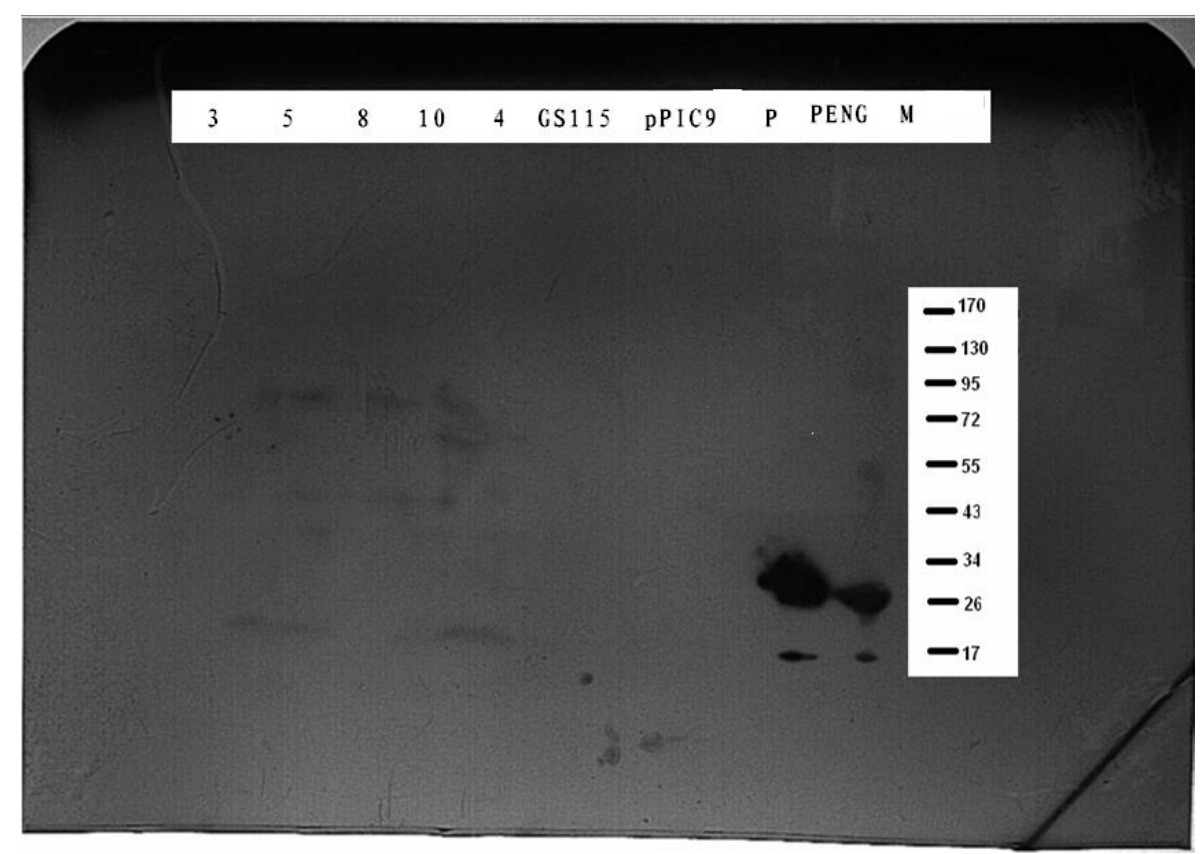

Figure 4. Western blot analysis of porcine BPI expression in yeast GS115. Lane M represents protein marker; lanes $3,5,8,10,4$ represent recombinant porcine BPI protein; P represents positive control.

(calculated MW: approximately $52 \mathrm{kDa}$ ) (Figure 5). Similar analysis of cell lysates and supernatants were performed at day 6 post-transfection (Figure 6). Approximately $10 \mu \mathrm{g}$ protein was obtained from $100 \mathrm{~mL}$ cell culture. 


\section{A. SDS-PAGE}

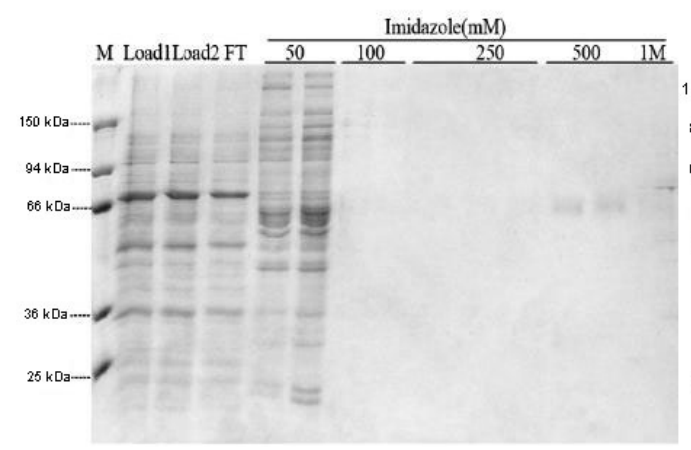

B. Western blot

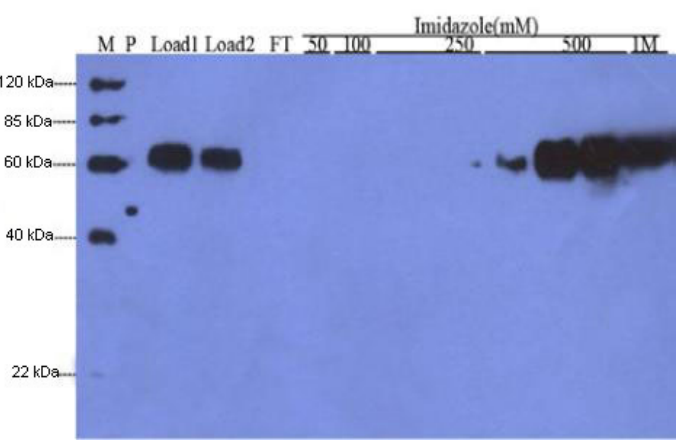

Figure 5. SDS-PAGE and Western blot analysis of BPI in HEK293 cells. Lane M: Protein marker; lane 1: Cultured medium after centrifugation; lane 2: Cultured medium after filtration; lane FT: Flow through; lane P: Multiple-tag protein (GenScript, Cat.No.M0101), as a positive control.

\section{SDS-PAGE}

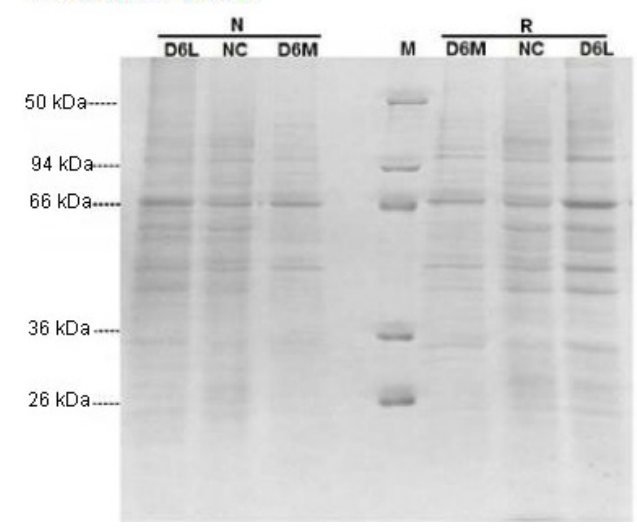

\section{Western blot}

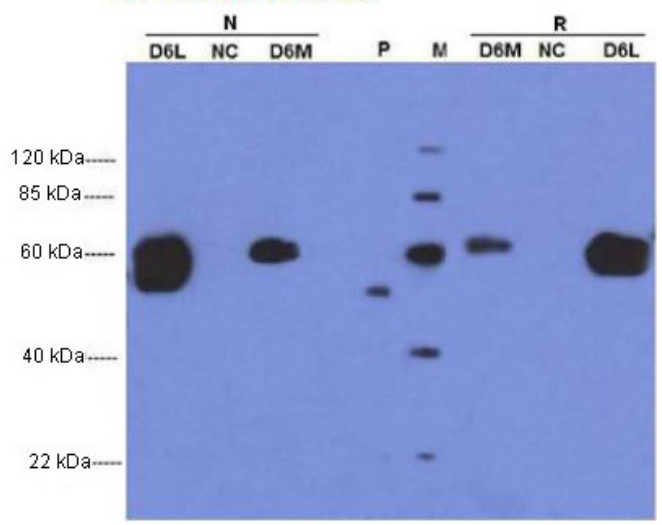

Figure 6. SDS-PAGE and Western blot analysis of BPI protein expression in HEK293 cells at day 6 posttransfection. Lane $M$ : protein marker; lane $N C$ : untransfected cell lysate; lane $N / D 6 L$ : cultured lysate at day 6 post-transfection under non-reduced conditions; lane N/D6M: cell culture medium at day 6 post-transfection under non-reduced conditions; lane $R / D 6 L$ : cultured lysate from day 6 post-transfection under reduced conditions; lane $R / D 6 M$ : cell culture medium from day 6 post-transfection under reduced conditions; lane $P$ : multiple-tag protein (GenScript, Cat.No.M0101), as positive control.

\section{Expression of BPI protein in $E$. coli F18-resistant and susceptible individuals}

Expression of the recombinant porcine BPI protein was detected as a band of approximately $53 \mathrm{kDa}$ in the duodenum of E. coli F18-resistant and susceptible Sutai pigs by Western blot analysis. The results showed markedly higher expression of the BPI protein in E. coli F18-resistant group compared with that detected in the E. coli F18-susceptible group (Figure 7). 

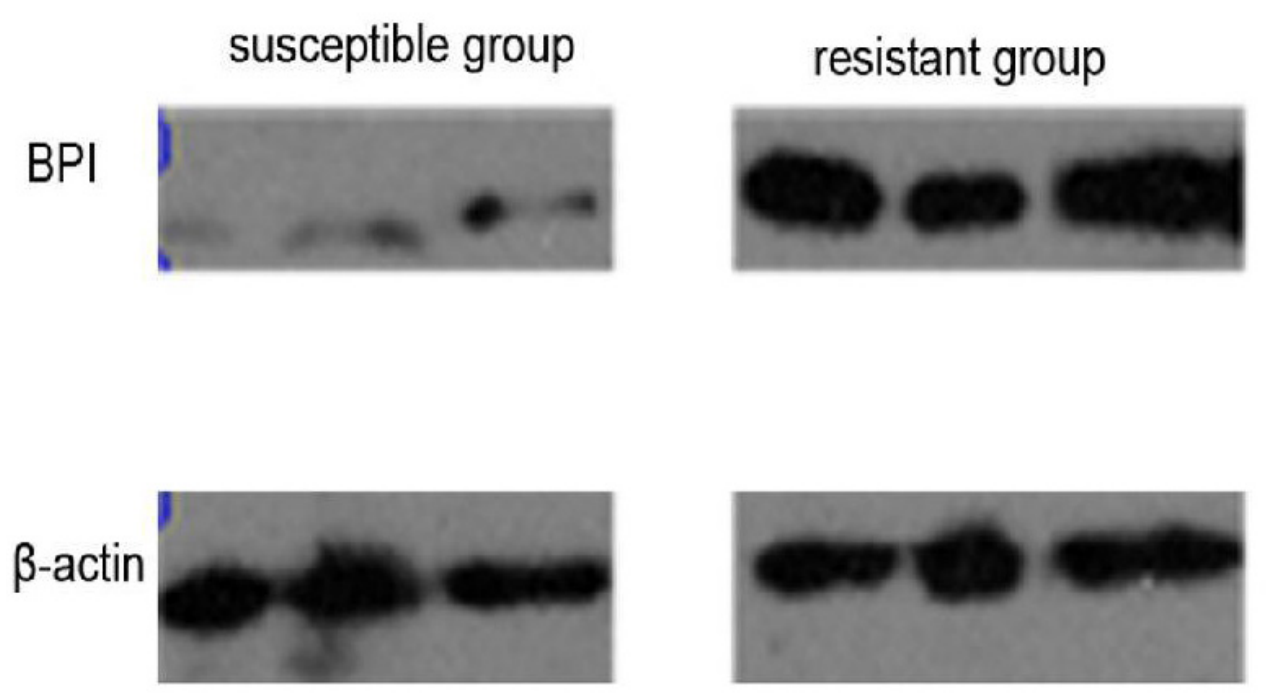

Figure 7. Western blot analysis of the differential expression of BPI protein in the duodenum of Escherichia coli F18-resistant and susceptible piglets $(\mathrm{N}=3$ per group).

\section{DISCUSSION}

BPI protein is a type of cationic antimicrobial glycoprotein secreted by neutrophilic granulocytes. BPI protein possesses bacteriostatic capacity against GNB, such as E. coli, Salmonella, Pseudomonas aeruginosa, Proteus vulgaris, Shigella dysenteriae, and Neisseria, although there are differences in the sensitivity to BPI among different bacterial strains. Furthermore, high concentrations of BPI can damage Gram-positive bacteria and eukaryotic cells (Elsbach, 1994). In addition, BPI neutralizes endotoxins and possesses antifungal activity. Little et al. (1994) reported that BPI combined with and neutralized heparin, indicates its capacity to inhibit angiogenesis. Their study also identified 3 structural domains of rBPI23 that were all related to biological activity, with the third domain (residues 148-161) possessing strong antifungal activity in vivo (Appenzeller et al., 1996). BPI had been identified as a new generation of protein drugs with the capacity for highly efficient sterilization and endotoxin neutralization, indicating its potential for applications involved in the resistance to microbial infection.

To date, systematic theoretical and applied studies on native and recombinant forms of BPI protein have been carried out. In addition to applications for treating GNB infectious diseases, series of BPI-derived peptides (such as antifungal drugs, anti-angiogenesis drugs, etc.) have been developed. BPI has been shown to be secreted in both in vitro and in vivo models using an adenoviral system for the delivery of BPI (Alexander et al., 2004). Secretory BPI protein effectively neutralizes lipopolysaccharide and reduces the generation of the inflammatory cytokine tumor necrosis factor- $\alpha$ and macrophage inflammatory protein-2. Zheng et al. (1999) investigated the molecular design, selection, and evaluation of the BPI structure/ function simulation peptide, resulting in the identification of a simulated peptide molecule possessing strong sterilization and endotoxin neutralization functions (Zheng et al., 1999). Currently, recombinant BPI-21 and BPI-23 have been approved for phase III clinical trials 
by the United States Food and Drug Administration. Further studies confirmed that rBPI21, rBPI23, and BPI-derived peptides all possess anti-angiogenic activity.

Recombinant BPI protein shows promise for clinical application. Therefore, in this study, BPI prokaryotic, eukaryotic, and yeast expression vectors were successfully constructed to identify a suitable system for the expression of recombinant porcine BPI. Expression of the target protein was not detected in the prokaryotic and yeast expression systems despite optimization of the expression conditions in E. coli. However, expression of the protein was detected in 293-6E cell culture supernatants in the eukaryotic expression system. Expression of porcine BPI has also been reported in Chinese hamster ovary cells (Xu et al., 2001a,b; Ma et al., 2001). These observations indicate the feasibility of using this eukaryotic expression system for bulk preparation of recombinant porcine BPI protein.

E. coli $\mathrm{F} 18$ is the primary GNB in the gut and the primary pathogen leading to diarrhea and edema disease in weaned piglets. In previous studies, Sutai pig resource populations were established carrying either the ETEC F18-resistant AA genotype or the ETEC F18-sensitive AG or GG genotypes. The display of functional adhesin through the type V secretion system was combined with receptor binding experiments to further analyze and verify the resistance and sensitivity to the ETEC F18 strain among these pig resource populations (Wu et al., 2007). Using these populations, we analyzed the expression level of the porcine BPI gene in vivo. Ye et al. (2011) analyzed the differential expression between E. coli F18-resistant and sensitive piglets using real-time PCR. The results showed that expression of BPI gene was extremely low or undetectable in various tissues, including the heart, liver, spleen, lung, kidney, stomach, muscle, thymus, and lymph nodes. In contrast, significantly high levels of BPI mRNA expression were detected in the duodenum and jejunum, with significant higher levels in resistant animals compared to in sensitive individuals. Zhu et al. (2011) also used real-time PCR to analyze the expression of the BPI gene in post-weaning Yorkshire, Sutai (E. coli F18-resistant population) and Meishan piglets. The results of this study showed that expression of the BPI gene in the duodenum and jejunum of Sutai piglets was significantly higher than that in Yorkshire and Meishan piglets. In a previous study, we analyzed BPI gene expression in Sutai piglets over a time period ranging from newborns to post-weaning days $8,18,30$, and 35 (Zhu et al., 2013). There were significant differences between 35-day expression and expression at other developmental stages in the duodenum, with the greatest vulnerability to E. coli F18 infection detected at 35 days of age. Therefore, previous studies revealed an association between the expression level of the porcine BPI gene and susceptibility to E. coli F18 strains. However, previous studies have mainly analyzed mRNA expression using real-time PCR, which does not determine the level of BPI protein expression. In this study, we further analyzed the correlation between BPI protein expression and E. coli F18 sensitivity by Western blotting. The expression level of the BPI protein in the E. coli F18-resistant group was significantly higher than that in the sensitive group. This study not only confirmed the conclusions of previous studies, but also suggested that $B P I$ protein expression is associated with the susceptibility or resistance to intestinal $E$. coli $\mathrm{F} 18$ infection, with increased intestinal $B P I$ protein expression associated with resistance to $E$. coli $\mathrm{F} 18$ infection.

\section{CONCLUSIONS}

In this study, we successfully constructed prokaryotic, eukaryotic, and yeast expression vectors for the porcine BPI protein. While the prokaryotic and yeast expression sys- 
tems failed to produce the target protein, recombinant porcine BPI protein was detected in the culture supernatant of the 293-6E cell eukaryotic expression system. These observations indicated strong bacteriostatic activity of the BPI protein, in addition to the potential for using the eukaryotic expression system for large-scale production of recombinant porcine BPI recombinant protein.

In addition, using $E$. coli $\mathrm{F} 18$-resistant and -sensitive piglets as an experimental model, we observed significantly higher expression of the BPI protein in the E. coli F18-resistant group compared with that in the $E$. coli $\mathrm{F} 18$-sensitive group. This indicates that higher expression of BPI is associated with the resistance to $E$. coli F18 infection. The findings of this study provide a basis for further investigations into the development of a drug designed to confer resistance to $E$. coli $\mathrm{F} 18$ in weaning piglets.

\section{ACKNOWLEDGMENTS}

Research supported by grants from the National Natural Science Funds (\#31372285 and \#31172183), the Natural Science Foundation of the Jiangsu Higher Education Institutions of China (\#14KJA230003), the Genetically Modified Organisms Technology Major Project (\#2014ZX08006-001B), and the Priority Academic Program Development of Jiangsu Higher Education Institutions.

\section{REFERENCES}

Akin H, Tahan G, Türe F, Eren F, et al. (2011). Association between bactericidal/permeability increasing protein (BPI) gene polymorphism (Lys216Glu) and inflammatory bowel disease. J. Crohns Colitis 1: 14-18.

Alexander S, Bramson J, Foley R and Zhou X (2004). Protection from endotoxemia by adenoviral-mediated gene transfer of human bactericidal/permeability-increasing protein. Blood 103: 93-99.

Alwan A, Designan T, Sullivan MO, Kelly J, et al. (1998). Quantitative assay of Salmonella adherence to intestinal epithelial cells: a new method for assessing novel intervention products. J. Microbiol. Meth. 33: 163-170.

Appenzeller L, Lim E, Wong P, Fadem M, et al. (1996). In vivo fungicidal activity of optimized domain III peptides derived from bactericidal/permeability-increasing protein (BPI). In: 36th Interscience Conference on Antimicrobial Agents and Chemotherapy. Washington, DC: American Society for Microbiology 132.

Bao WB, Ye L, Zhu J, Pan ZY, et al. (2012). Microarray analysis of differential gene expression in sensitive and resistant pig to Escherichia coli F18. Anim. Genet. 43: 525-534.

Canny G and Levy O (2008). Bactericidal/permeability -increasing protein (BPI) and BPI homologs at mucosal sites. Trends Immunol. 29: 541-547.

Elsbach P (1994). Bactericidal/permeability-increasing protein in host defense against Gram-negative bacteria and endotoxin. Antimicrobial Peptides, Ciba Foundation Symposium 186: 176-189.

Elsbach P (1998). The bactericidal/permeability-increasing protein (BPI) in antibacterial host defense. J. Leukoc. Biol. 64: 14-18.

Gavit P and Better M (2000). Production of antifungal recombinant peptides in Escherichia coli. J. Biotechnol. 79: 127-136.

Iovine NM, Elsbach P and Weiss J (1997). An opsonic function of the neutrophil bactericidal/ permeability increasing protein depends on both its N- and C-terminal domains. Proc. Natl. Acad. Sci. U.S.A. 94: 10973-10978.

Little RG, Kelner DN, Lim E, Burke DJ, et al. (1994). Functional domains of recombinant bactericidal/permeability increasing protein $\left(\mathrm{rBPl}_{23}\right)$. J. Biol. Chem. 269: 1865-1872.

Ma YY, Ma WY, Yu WB, Yin W, et al. (2001). The cDNA cloning and expression of Fab-BPI fusion protein in CHO cell line. J. Mol. Cell. Immunol. 17: 470-472.

Ooi CE, Weiss J, Doerfler ME and Elsbach P (1991). Endotoxin-neutralizing properties of the $25 \mathrm{kD} \mathrm{N}$-terminal fragment and a newly isolated $30 \mathrm{kD} \mathrm{C}$-terminal fragment of the $55-60 \mathrm{kD}$ bactericidal/permeability-increasing protein of human neutrophils. J. Exp. Med. 174: 649-655.

Tuggle CK, Stabel TJ, Shi XW and Mellencamp MA (2003). Genetic markers for improved disease resistance in animals (BPI). United States, Kind Code: A1, Patent Application: 20030104424, Nov 25. 
Weiss J, Elsbach P, Olsson I and Odeberg H (1978). Purification and characterization of a potent bactericidal and membrane active protein from the granules of human polymorphonuclear leukocytes. J. Biol. Chem. 253: 2664-2672.

Wu SL, Yuan ZW, Ju HP, Huang XG, et al. (2007). Polymorphisms of the FUT1 gene M307 locus in post-weaning Sutai breed piglet and resistance to F18 fimbrial Escherichia coli in vitro. Chin. J. Prev. Vet. Med. 29: 783-787.

Xu JJ, Xu J and Wang HT (2001a). Gene cloning and expression of human bactericcdal/permeability-increasing protein. Wei Sheng Wu Xue Bao 46: 669-673.

$\mathrm{Xu} \mathrm{JJ}, \mathrm{Xu}$ J, Tong YG and Wang HT (2001b). Expression of recombinant BPI23-Fc $\gamma 1$ fusion protein in CHO cells. Chin. J. Biotechnol. 17: 587-589.

Ye L, Zi C, Liu L, Zhu J, et al. (2011). Study on the relationship between the expression of BPI gene and Escherichia coli F18 infection in piglets. Yi Chuan 33: 1225-1230.

Zheng J, Zhou H, Xia PY, Qing XJ, et al. (1999). In vivo antiendotoxin effect of simulation peptide of rabbit bactericidal/ permeability-increasing protein (BPI). Sichuan J. Physiol. Sci. 21: 16.

Zhou H, Zheng J and Xiao GX (1992). Effect of porcine Bactericidal/permeability-increasing protein on Gram-negative bacteria. Chin. J. Infect. Dis. 17: 47-48.

Zhou H, Yuan JC, Zhou LX, Zheng J, et al. (1999). The function of neutralizing endotoxin of bactericidal/permeability increasing protein (BPI) in vitro. National Medical J. 79: 304-305.

Zhou H, Zheng J, Qing XJ and Xiao GX (2002). Enhancement of anti-human bactericidal/permeability increasing protein (BPI) antibodies on bio-activities of porcine BPI in vitro. Acta Academiae Medicinae Militaris Tertiae 24: 76-78.

Zhu J, Zi C, Su XM, Liu L, et al. (2011). Expression differentiation of BPI gene in post-weaning piglets of Yorkshire, Sutai and Meishan breeds. J. Anim. Vet. Adv. 10: 3155-3160.

Zhu J, Zi C, Wu ZC, Liu L, et al. (2013). Age-dependent expression of the BPI gene in Sutai piglets. Genet. Mol. Res. 12: 2120-2126. 